JPHSR 2013, ••: ••-•• () 2013 Royal Pharmaceutical Society

Received September 24, 2012 Accepted April 12, 2013 DOI 10.1111/jphs.12023 ISSN $1759-8885$
Correspondence: Flavia Bruno, Via Balzaretti, 9, 20133 Milano MI, Italy.

E-mail: flavia.bruno@unimi.it

\section{Community pharmacists and stress at the counter: qualitative exploratory initial Italian research}

\author{
Egidio Mojaa ${ }^{a}$ Paola Minghetti ${ }^{b}$, Luisa Vercellesic, Elena lapichino ${ }^{a}$, \\ Stefania Anania ${ }^{a}$, Martina Laccisaglia ${ }^{d}$ and Flavia Brunoc
}

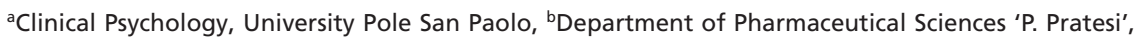
'Centre of Studies on Drug Communication, 'Department of Pharmacological and Biomolecular Sciences, University of Studies of Milan, Milan, Italy

\begin{abstract}
Objectives Community pharmacists are the most accessible healthcare professionals. They interact with a large number of patients who often show emotional concern. Their stressful and complex role is widely acknowledged, nonetheless research on their personal life is practically non-existent. Thus, we set out to qualitatively explore the emotional perceptions of pharmacists.

Methods Twenty experienced pharmacists were interviewed by a psychologist and a pharmacist. Data were analysed using an interpretative phenomenological analysis and coded using NVivo qualitative data analysis software.

Key findings Interviewed pharmacists admitted to different types of difficulties, mostly arising from a series of small, standalone episodes. Their stories describe intrinsic difficulties with their practice, rather than personal experiences. This study reveals the complexity of a community pharmacists' role - divided into health and hygiene advice, counselling and empathic relationships, and entrepreneurship. An important obstacle is the integration and concomitant implementation of these components. Endogenous factors such as personal experiences, personality and academic background, and exogenous factors such as social status of the patient population, legal and administrative issues and location, all affect pharmacists' ability to cope with their role.

Conclusions Improving health care can be obtained by incorporating community pharmacists into the healthcare team. Technical training is important in order to improve relationship skills and personal coping strategies. Further studies are recommended to provide a simple tool for measuring aspects of the personal and emotional aspects (inner life) of a community pharmacist.
\end{abstract}

Keywords inner life; patient-centred medicine; pharmacist psychology; professional burnout; qualitative research

\section{Introduction}

Pharmacists are the most accessible healthcare professionals whose daily interactions with a large number of patients results in some form of emotional involvement. Thus, even if drug dispensing and counselling are still their major tasks, the role is more complex in nature.

Italy has population of over 60 million people, and there are 17796 community pharmacies, an average of one pharmacy every 3374 persons, a ratio very close to the European average (3323). ${ }^{[1]}$ Community pharmacists serve about three million patients daily. ${ }^{[2]}$ In addition to the interaction with a diverse patient population, pharmacists are faced with many challenges. Multiple factors contribute to the complexity of their role and influence their professional behaviour and personal life. Several of these factors include increased demands on pharmacists' time for administrative reasons, security issues, ethical responsibilities, increased interactions with other healthcare providers and the delivery of extended services. ${ }^{[3,4]}$ An individual's level of frustration increases as the number of stressors in a situation increases, thus increasing the likelihood of emotional reactions. ${ }^{[5]}$

The direct patient care provided by pharmacists has favourable effects for patients. It builds a relationship and facilitates counselling which in turn may improve patient compliance to treatments and clinical outcomes. Indeed, a recent meta-analysis on 298 studies has shown favourable results in therapeutic and safety outcomes. Haemoglobin A1c, low-density 
lipoprotein cholesterol, blood pressure and adverse drug events are some examples favouring pharmacists' direct patient care over comparative services $(P<0.05)$. The same meta-analysis shows that medication adherence, patients' knowledge, and quality of life all support the involvement of pharmacists' inpatient care $(P<0.05){ }^{[6]}$

On the other hand, the continuous interactions with distressed patients and involvement in stressful situations may have a negative impact on pharmacists. Stressful professional working conditions and their empathic approach to patients have an impact on their personal life, the so-called inner life, that is the sphere representing emotional perceptions and ideas and professional distress. ${ }^{[7]}$

An increasing number of studies examine the negative impact that witnessing or learning of trauma experienced by their patients has on clinicians. Many studies have been published since the 1970s on the personal lives of health operators, such as doctors and nurses, focusing on their relationship with aggressive and terminal patients. ${ }^{[8]}$ This vicarious traumatisation has been described with various terms: secondary victimisation, secondary survival, emotional contagion, counter-transference, burnout and compassion fatigue. The use of empathy is a common denominator of these processes, and emotional detachment does not protect health professionals from distress and burnout. ${ }^{[9]}$ In spite of the recognized stressful role of the pharmacist's practice, an extensive searching in PubMed using all above concepts has led to very few publications which focus on their emotional perceptions and experiences. Thus, the aim of this study was to qualitatively assess the inner life of pharmacists. This was investigated by the thorough analysis of interviews in order to map their feelings and difficulties faced during their daily routine.

\section{Methods}

\section{Sampling}

E-mailed invitations were used initially to recruit a random sample population of 250 pharmacists belonging to the professional charter of Milan and Lodi (northern Italy). Due to very little positive feedback, we turned to enrolling 20 pharmacists with a convenience inclusion. Enrolled pharmacists included men and women, less than 60 years of age, and with a professional experience of at least 3 years.

\section{Study design, data collection and analysis}

We implemented an exploratory, qualitative approach according to an interpretative phenomenological analysis (IPA). ${ }^{[0]}$
This allows for the researcher to focus on the way in which interviewees tell their stories, and how they perceive themselves and carry out their job. This method facilitates the exploration of the emotional perceptions and personal life of the pharmacist.

Semi-structured interviews were implemented since they represented the best method of obtaining the information required for the study. They made it easier for interviewees to use their own definitions of the issues, thus allowing the researcher to pursue the topics of interest. ${ }^{[11]}$ Interviews were conducted by two researchers in pairs, a psychologist and a pharmacist. They were carried out in a suitable, quiet room at the Centre of Studies for Drug Communication at the University of Milan, Italy.

All interviews began by an open predefined question asking for a description of experiences at the counter that caused them difficulties. There were also three predefined open questions that could be asked at any time during the interview: what emotions were being felt, what situations were commonly experienced on the job (sporadic or repeated), and what advice could be provided to young pharmacists for their future profession. Researchers rarely interrupted the interview; they could ask for clarifications or rekindle the memory of emotional aspects.

This study was approved by the Ethical Committee of the University Pole San Paolo, University of Milan. All interviews were tape-recorded with permission and transcribed.

The preliminary results underwent a team discussion. In accordance with IPA, the strategy followed consisted in (1) careful reading of the transcripts to grasp an initial, general understanding and to structure pre-concepts; (2) defining aspects emerging from the interviewees' experiences; (3) categorizing contents into coded units; and (4) generalizing concepts that could be extrapolated from each coded unit. The emerging categories were discussed and agreed within the research team. The data were re-examined after computer coding using the qualitative data analysis NVivo test (QSR International, Doncaster, Victoria, Australia (http://www. qsrinternational.com; accessed February 2011)) and quantify the categories. The NVivo analysis was carried out after the completion of all the interviews.

\section{Results \\ Demographic data}

Interviews were carried out in 2010; they lasted an average of $21 \mathrm{~min}$, ranging from $11.2-39.3 \mathrm{~min}$. Table 1 summarizes the

Table 1 Demographic data of the interviewees

Gender, $n,(\%)$

Location of the pharmacy, $n,(\%)$

Type of pharmacy, $n,(\%)$

Role in the pharmacy, $n,(\%)$

Mean age in years

Mean experience in the profession in years

Mean duration of the interview in minutes
Men, 9, (45\%)

(Urban) Milan, Italy, 10, (50\%)

*Municipal, 3, (15\%)

Owner, $15,(75 \%)$

48 (minimum 33; maximum 64; median 47)

27 (minimum 3; maximum 34; median 20)

20.3 (minimum 11.2; maximum 39.3; median 19.5)
Women, 11, (55\%)

Rural areas, $10(50 \%)$

Private, 17, (85\%)

Employee, 5, (25\%)

*Public pharmacies owned by the county of Milan. 
demographic data of the interviewed sample, which was obtained from the anonymous questionnaire filled out by the interviewees. Consent was obtained to transcribe, analyse and disseminate findings and the conclusion obtained.

\section{Findings}

Table 2 shows all of the categories identified from the interviews. The preliminary coding of transcript interviews has led to the identification of four main categories: (1) personal relationships; (2) comments about pharmacists' academic curriculum and ongoing education; (3) job-related difficulties; and (4) feelings. Table 3 shows categories grouped according to several characteristics of the interviewed pharmacists.

A detailed analysis of the differences observed between the categories is shown in Table 3 and is briefly discussed below:

(1) personal relationships:

- pharmacists in municipal pharmacies $(n=3)$ show less attention to personal relationships than those working in private pharmacies $(n=17)(66.7 \%$ versus $100 \%)$;

- another difference emerging between municipal and private pharmacists concerns a higher degree of personal involvement expressed in terms of feelings (66.7\% versus $47.1 \%$ ); this difference could be related to the generally greater attention shown by these particular pharmacists to patients - a personality trait that might have contributed to their reasons for working in a public rather than a private pharmacy;

- doctors appear to be central in the pharmacist's profession and frequently pharmacists act as mediators of their prescriptions $(60 \%)$ even if direct contact is low $(10 \%)$;

(2) academic curriculum and continuing education:

- women appear to pay more attention to academic accomplishments, since they readily have comments and suggestions for improvement $(81.8 \%$ versus $55.6 \%$ men);

- this difference is similar for pharmacists in municipal pharmacies $(100 \%$ versus $64.7 \%$ in privately owned pharmacies);

(3) job-related difficulties:

- difficulties reported by employees (60\% versus $53.3 \%$ owners) are more frequent, possibly due to the lack of independence within their job;

(4) feelings:

- only $50 \%$ of interviewees discussed their feelings, which are mainly self referential and negative; frustration is the commonest (30\%); only $15 \%$ refer empathy towards patients;

- difficulties and feelings appear to be unrelated to the number of years' experience in the profession (19.2 and 23.6 years respectively, mean 20.7 years).

Table 2 Main categories and themes emerging from interviews with 20 pharmacists, according to the inductive analysis

\begin{tabular}{|c|c|c|}
\hline $\begin{array}{l}\text { Categories, } n(\% \text { of } \\
20 \text { interviewees })\end{array}$ & Themes & $\begin{array}{l}n(\%) \text { expressed on the total } \\
\text { of the relevant category }\end{array}$ \\
\hline \multirow{5}{*}{$\begin{array}{l}\text { Human relationships } \\
\quad n=19 \text { (95) }\end{array}$} & Mediation between patient and doctor & $12(60)$ \\
\hline & Mediation between patient and psychologist & $6(30)$ \\
\hline & Patient counselling and education & $5(25)$ \\
\hline & Negative assessment by stakeholders & $3(15)$ \\
\hline & Collaboration with doctors & $2(10)$ \\
\hline \multirow{2}{*}{$\begin{array}{l}\text { Comments regarding academic curriculum } \\
n=14 \text { (70) }\end{array}$} & Need to improve relationship skills and technical competence & $10(50)$ \\
\hline & Need to improve emotional and linguistic abilities & $6(30)$ \\
\hline \multirow{4}{*}{$\begin{array}{l}\text { Difficulties } \\
\quad n=11(55)\end{array}$} & Bureaucracy and procedures & $5(25)$ \\
\hline & Loss of professional competencies & $5(25)$ \\
\hline & Limited decision making & $3(15)$ \\
\hline & Robberies & $3(15)$ \\
\hline \multirow{4}{*}{$\begin{array}{l}\text { Feelings } \\
\quad n=10(50)\end{array}$} & Frustration & $6(30)$ \\
\hline & Empathy towards patients & $3(15)$ \\
\hline & Mental burnout & $2(10)$ \\
\hline & Lack of comprehension of the role by stakeholders & $2(10)$ \\
\hline
\end{tabular}

Table 3 Categories emerging from the interviews according to pharmacists' characteristics

\begin{tabular}{lcccc}
\hline Characteristics (entire sample) & Human relationships & $\begin{array}{c}\text { Comments regarding } \\
\text { academic curriculum }\end{array}$ & Difficulties & Feelings \\
\hline Mean age (48 years) & 49 years & 50 years & 48.5 years & 52.3 years \\
Mean experience in the profession (20.7 years) & 21.6 years & 22.7 years & 19.2 years & 23.6 years \\
Male $(n=9)$ & $n=8(88.9 \%)$ & $n=5(55.6 \%)$ & $n=5(55.6 \%)$ & $n=2(22,2 \%)$ \\
Female $(n=11)$ & $n=11(100 \%)$ & $n=9(81.8 \%)$ & $n=6(54.5 \%)$ & $n=8(72,7 \%)$ \\
Municipal pharmacies $(n=3)$ & $n=2(66.7 \%)$ & $n=3(100 \%)$ & $n=2(66.7 \%)$ & $n=2(66.7 \%)$ \\
Private pharmacies $(n=17)$ & $n=17(100 \%)$ & $n=11(64.7 \%)$ & $n=9(52.9 \%)$ & $n=8(47.1 \%)$ \\
Pharmacy owners $(n=15)$ & $n=15(100 \%)$ & $n=11(73.3 \%)$ & $n=8(53.3 \%)$ & $n=8(53.3 \%)$ \\
Employees $(n=5)$ & $n=4(80 \%)$ & $n=3(60 \%)$ & $n=3(60 \%)$ & $n=2(40 \%)$ \\
\hline
\end{tabular}


Table 4 Results according to computer-assisted NVivo analysis

\begin{tabular}{lcc}
\hline Emerging factors and classification & Interviewees referring factors, $\boldsymbol{n}(\boldsymbol{\%})$ & Coded references, $\boldsymbol{n}$ (mean for interviewees) \\
\hline Job roles & $20(100)$ & $135(6.8)$ \\
\hline $\begin{array}{l}\text { Integration of entrepreneur role with sanitary and } \\
\quad \text { counselling roles }\end{array}$ & $17(85)$ & $65(3.8)$ \\
$\begin{array}{l}\text { Integration of health and hygiene advice and } \\
\quad \text { counselling roles }\end{array}$ & $16(80)$ & $70(4.4)$ \\
\hline Endogenous factors & & $n=70(4.1)$ \\
\hline Experience & $n=17(85)$ & $44(2.9)$ \\
Character & $15(75)$ & $18(2.3)$ \\
Academic curriculum & $8(40)$ & $8(2.0)$ \\
\hline Exogenous factors & $4(20)$ & $n=62(4.4)$ \\
\hline Social setting & $n=14(70)$ & $32(2.7)$ \\
Legislation and bureaucracy & $12(60)$ & $25(2.5)$ \\
Location & $10(50)$ & $5(1.3)$ \\
\hline
\end{tabular}

Table 5 Characteristics of interviewees for each factor, deriving from computer-assisted NVivo analysis

\begin{tabular}{lccc}
\hline Characteristics (entire sample) & Professional roles & Endogenous factors & Exogenous factors \\
\hline Mean age (48 years) & 48 years & 49.4 years & 47.1 years \\
Mean experience in the profession (20.7 years) & 20.7 years & 21.9 years & 20.5 years \\
Male $(n=9)$ & $n=9(100 \%)$ & $n=6(66.7 \%)$ & $n=6(66.7 \%)$ \\
Female $(n=11)$ & $n=11(100 \%)$ & $n=11(100 \%)$ & $n=8(72.7 \%)$ \\
Municipal pharmacies $(n=3)$ & $n=3(100)$ & $n=3(100 \%)$ & $n=2(66.7 \%)$ \\
Private pharmacies $(n=17)$ & $n=17(100 \%)$ & $n=14(82.4 \%)$ & $n=12(70.6 \%)$ \\
Pharmacy owners $(n=15)$ & $n=15(100 \%)$ & $n=13(86.7 \%)$ & $n=10(53.3 \%)$ \\
Employees $(n=5)$ & $n=5(100 \%)$ & $n=4(80 \%)$ & $n=4(80 \%)$ \\
\hline
\end{tabular}

- during their interview, $72.7 \%$ of women versus $22.2 \%$ of men referred to their feelings; this difference could be the reason why the women tended to provide comments on academic background and curriculum.

Table 4 shows the details of the findings as supported and confirmed by the computer-assisted NVivo analysis as well as the number of references coded for each theme. All interviewees discussed their job role in the practice, $85 \%$ and $70 \%$ of interviewees described endogenous and exogenous factors respectively. Table 5 shows the same results analysed according to the demographic characteristics of the interviewees.

Interviewed community pharmacists acknowledged general and daily difficulties. The occurrence of a series of small independent episodes was a daily obstacle. Their stories mainly describe intrinsic continuing difficulties in their practice rather than individual negative episodes.

\section{Job role}

The pharmacist's job role appears to be split into three different parts. Each part requires a complex integration.

(1) health and hygiene advice;

(2) patient counselling, relationships and empathy;

(3) entrepreneurship.

These three aspects underline different objectives and require specific competences and skills. They are complementary and overlapping, but sometimes diverge in focus. Tension and major professional difficulties can emerge in the relationship with patients in situations in which the three professional roles should be played out simultaneously, but in fact a final decision on the prevailing one is needed.

\section{Health and hygiene advice}

Pharmacists consider the primary aim of their job as a service is to protect and care for the health and wellness of patients. In doing so they are required to adhere to strict laws on drug dispensing while at the same time providing information on drug activity, dosage, contraindications and safety. The most critical situation is when pharmacists' need to enforce restrictions on drug dispensing while maintaining a good relationship with patients. Often, conflicts emerge when patients ask for a drug but their prescriptions are incorrect, old or do not exist at all. This is particularly true in case of an emergency:

'... it is impossible for us to dispense drugs if the prescription is unavailable. if the emergency medical wards are not answering the phone or they do not visit patients at home... What can we do? Do we try to cope with the problem as quickly as possible or do we comply with the law?' F0104

Pharmacists have said that since they are easily accessible, they often receive inadequate requests falling outside their role. For 
example, patients often ask pharmacists to interpret the medical doctor's role. Specifically, pharmacists are often faced with interpreting clinical tests, requesting treatment without consulting the family doctor and having to give a diagnosis:

'A mother comes and says she has incorrectly used several lotions: "I have been in another pharmacy and they told me so and so" or "the doctor said this, the specialist said that'. How do you behave? You should avoid having a know-it-all approach, but it is important to tell the truth, the patient could simply get angry or even worse get hospitalized, this is a big issue.' F0101

Moreover, pharmacists have to respect the role of prescribers. The difficulties in the management of these boundaries are apparent from their stories:

'... mothers have come to me inquiring if the prescriptions for her child were amphetamines. . . . it is difficult to know what you are expected to say, or what you shouldn't say unless asked to do so ... we are often caught between the prescriber and the patient. This is the most critical issue.' F0101

\section{Patient counselling, relationships, empathy}

Educating and counselling patients in terms of psychophysical health prevention and promotion is a primary objective, which can be achieved mostly through an empathic relationship:

'Drug dispensing is not our sole responsibility ... Advancement in technology could allow for automated drug dispensing . . . and we would not be necessary. We have to understand and help patients with a problem. Our added value and gratification is through giving something more in terms of human relations and counselling.' F0112

This trait is not one that can just be handed over the pharmacy counter. It cannot be obtained through years of experience nor through education. However, it seems to be an intrinsic trait. Interestingly, pharmacists are often compared to other professions, such as psychologists and teachers, owing to their tendency to counsel patients and to develop personal relationships with them:

'We need to behave like psychologists, because we need to know who we are dealing with, and how to approach different situations. From my point of view, we are more and more personally involved, because from prescriptions we understand the disease.' F0110 'The pharmacist is the last link. . . In case of serious conditions it is clear that he/she is there and ready to register difficult psychological and emotional situations. Sometimes in my profession I felt more a confessor than a pharmacist.' F0109

The management of the pharmacist's feelings, along with that of the patient's personal life, is a difficulty encountered by pharmacists in their role as counsellors. The pharmacist feels the need to establish a trustworthy relationship with the patient. This is considered to be a job-related objective and a source of daily satisfaction, but is not devoid of difficulties:
'The most rewarding aspect of the profession is establishing a relationship with the patient. From a psychological point of view ... these are beautiful aspects, because they show you that your commitment is recognized by your patient.' F0112

The pharmacist has a tendency to be at the mercy of patients' needs and moods. This generates experiences which feature contradictory emotions. On the one hand, these experiences lead to personal satisfaction and professional enrichment, while on the other hand they require an investment of energy. This can lead to burnout and a sense of shortcoming:

‘. . sometimes I am really worn out. I have to constantly smile no matter what.' F0110

'In pharmacies, relationships are formed ... pharmacies are not fashion stores. Patients are concerned about their health and you have to deal with many of them daily, each with different personalities and moods: first you meet a grandmother who is happy for her new-born grandson ... The next patient has a relative which is terminally ill ... this means you have to shift from joy to discretion.' F0101

'To get into others' brains, to be empathic on the same wave length. I can be right and be perfect, but when I have to establish a relationship with a person, I have to leave my way of thinking and step into their shoes. This happens even with your peers as regards culture or language, if they are in a different mood.' F0101.

Moreover, it is often difficult to deal with terminally-ill patients:

'Sometimes people approach you who are completely unaware of the severity of their loved-one . . . it is rather indicative of the situation when they have a prescription for "pain therapy" . . it is difficult to cope with this situation.' N7

The counselling role is where patients' and pharmacists' personal lives meet. Pharmacists were asked which traits they possess for the management of these situations. They identified two factors. The first is their personality (intrinsic):

'I am always very involved in all the situations, it is part of my personality. Clearly this is subjective.' F0110

The second trait is years of experience in the job role:

'We can say that when you have finished your education, you know everything about the chemistry of drugs. In reality, you realize that you have to implement basic and essential science, but most of it is having to do with much more than science. The easy aspect is reading a prescription ... in the past when prescriptions were handwritten it was a bit more complicated. The hard part is when you are requested to give advice. Clearly, this generates anxiety for newcomers.' F0112

\section{Entrepreneurship}

In addition to their roles regarding hygiene advice and counselling, pharmacists need to play a role in the pharmacy as a business: 
'at the end it is a business and as in all businesses we need revenue. Otherwise, it is clear that if we don't sell the pharmacy will have to close.' F0109

However pharmacists have difficulties in carrying out this role, particularly at times when patients are able to perceive this and forget the relationship of trust that has been established through the pharmacist's advice on hygiene and provision of counselling.

'I have to pay attention to the economical aspect, I will not be a pharmacist, but an entrepreneur. Often, patients aren't satisfied unless they obtain a discount. This implies that I have to purchase products which allow me to grant discounts on products ... One day pharmacists will be salespersons, just like in a supermarket. . . . in my opinion we already are.' F0108

\section{The integration of job roles}

The need to integrate the three job roles gives rise to much difficulty. The competence in health issues sometimes does not coincide with the counselling role and may interfere with the economic requirements of the pharmacy. This leaves pharmacists in a continuing search of a satisfactory stance. The observation which underlines this concept is:

' . . if I have to pay attention only to an economical aspect, I will not be a pharmacist, but an entrepreneur ...' F0108

'You need to learn all aspects of the profession, but do a good job ... and this is tiresome because at night you have absorbed everybody's problems.' F0103

'I think that money is a second consideration. It should never be the starting point. It is a secondary aspect, which comes after having considered the relationship.' F0105

\section{Factors involved in the job role}

From analysis of the interviews it appears that the job role of pharmacists is subject to two more considerations: endogenous factors, such as experience, personality and skills, and academic curriculum, and exogenous factors, including the social status of the population addressed and the location of the pharmacy.

\section{Endogenous factors}

Education and experience in the field facilitate the job role. However, the pharmacist's personality also strongly affects it. Being educated and open-minded in facing the different requirements of patients, however, does not appear to suffice in order to protect pharmacists from emotional stress which leads to feelings of impotence and loneliness:

'We all have a specific background, our own culture which can help in problem solving, but only to a certain extent. We can study and reduce our limits, but still we are pharmacists only ... and we have to recognize this limit. F0101

Regarding empathy it is a matter of personal character.' F0101
'The experience is very important. if you have already addressed a lot of situations this fact helps you. With years this gives you a greater pliability, you can understand the best way to come to a solution.' F0101

\section{Exogenous and environmental factors}

Community pharmacists experience pressures which come from the patients' social class as well as from where the pharmacy is located:

'Our profession is the only one which allows everyone and anyone to get in touch with us; other health professionals have the possibility of filtering contacts, while the pharmacy is open to everybody at anytime.' F0117

The issue of legal and administrative requirements is common to all pharmacists. There is the need for continuing education on scientific and medical matters while facing the role as a filter to amend any errors from prescribers and clarify doubts in patients. The pharmacist's role is to protect health and this requires a compromise with legal and administrative rules:

'More than difficulties I would define several factors which make our profession impracticable. We are expected to read and understand two thousands things every day ... It gets pretty hard to follow all these. In my opinion it is becoming a profession which is not compatible with real life.' F0108

\section{Discussion}

This is an exploratory study aimed at examining the workrelated experiences of pharmacists in Italy. The low number of interviews, their restricted location, the large percentage of pharmacy owners and private pharmacies interviewed indicates that this is a preliminary study. Nonetheless interviews run in pairs by a psychologist and a pharmacist, and the use of the NVivo technique in the analysis of stories, provide a sound basis for understanding the inner life of pharmacists, and for a subsequent more extensive study in a field where no previous evidence was available. As expected, $100 \%$ interviewees have discussed issues in their job role. Eighty-five percent of them addressed endogenous elements while $70 \%$ discussed exogenous elements. The large percentages of issues is a strong indicator of the need for the provision of support to pharmacists in their daily activities at the counter and thereby limit their burnout.

The major factor distinguishing community pharmacists' experiences from those of other healthcare professionals, such as doctors and nurses, is their point of view. Pharmacists tend to see a series of continuing episodes, rather than sporadic episodes, which can lead to difficulties at the counter.

A second factor common to interviewed pharmacists is their multi-faceted role, which makes the profession particularly complex and may sometimes turn pharmacists' thoughts away from their first priority, namely the health of their patients. In fact, pharmacists are torn between health care, counselling and business obligations - all are elements which need to be mastered and deployed simultaneously. Protecting patients' health through counselling is considered the most 
important commitment in a pharmacists' practice. Mediation between patient and doctor is considered a key component of their relationship. Other commitments identified by most interviewees were the integration of the economic goals of the pharmacy (i.e. the entrepreneur role) and the need to keep a complementary and supporting role with prescribers. The large proportion of 'owners' in this study may have affected this view. Noteworthy are the contradictory goals of the three principal commitments within a pharmacist's practice. Regarding business obligations, stories from pharmacy owners and employees highlight different priorities, most likely because of economic pressure and the need to acquire and retain customers.

Key, endogenous factors affecting $75 \%$ of the performance of the pharmacists interviewed were competencies and skills such as empathy, communication and relationships with their patients. This was further supported by the finding that only $20 \%$ of interviewees considered academic qualifications to be an important asset. This underlines the need to integrate academic background with the delivery of continuing education with appropriate subjects, and is in agreement with previous studies. ${ }^{[12]}$ With regard to relationships, the lack of specific preparation focusing on relationship skills and management has been specifically underlined by our interviewees. The capacity to establish a sound relationship is a key element in patient-oriented care. Counselling is favoured by being able to develop an empathic relationship with patients, which in turn contributes to increasing patients' awareness of positive effects on health. ${ }^{[13]}$

A series of exogenous elements such as legislation and administrative issues (a common denominator in all pharmacies), and social environment have been traced in $70 \%$ of interviewees' stories. Due to the unbendable nature of these elements, specific training on coping strategies should be implemented. ${ }^{[3]}$

The development of empathic communication and relationship skills should help establish a profitable relationship between pharmacists and patients. It will also allow for an exchange of information, which is necessary to improve patients' health and well-being. Empathic, patient-oriented care has been shown to be associated with better health outcomes. Seeking to understand a patient with a life-threatening disease, and actively involving patients in their care fosters trust, thereby encouraging the patient to reveal important diagnostic information and be compliant with treatment. ${ }^{[7]}$ Full incorporation of community pharmacists into the healthcare team in direct patient care is a valuable solution to improve health care. ${ }^{[6]}$ Thus, as suggested by our interviewees, it is important to train pharmacists to exploit the development of relationship skills and personal, emotional coping strategies. This result supports previous evidence from the literature $^{[13-15]}$ and must become a priority for pharmacy academia.

It is interesting to note that in Italy, as opposed to other Western countries, the academic curriculum for pharmacists does not include teaching on communication, psychology, etc. Thus it is important to recognize these issues in the role, support pharmacists adequately, provide suitable continuing medical education and lobby to have such teaching included in the Italian pharmacy academic curriculum.

\section{Conclusion}

In view of the value of pharmacists in the community, and the need to remove obstacles within their profession so that they can provide the optimum service, further studies in this field are recommended. A user-friendly tool should be developed to measure aspects of the inner life of community pharmacists. Specifically, a focus should be placed on the daily obstacles they encounter. ${ }^{[4]}$ On the basis of the stories emerging from exploratory research we are devising a questionnaire which will be administered nationwide.

\section{Declarations}

\section{Conflict of interest}

The Author(s) declare(s) that they have no conflicts of interest to disclose.

\section{Funding}

This project was partially funded by a grant from the Charter of Pharmacists of Milan and Lodi, Italy.

\section{Acknowledgements}

The Authors thank Dr Antonietta Silini, PhD, for her linguistic revision and editing.

\section{Authors' contributions}

E. Moja, P. Minghetti and F. Bruno were mainly involved in the study design; E. Iapichino, S. Anania, L. Vercellesi, and M. Laccisaglia did the data collection and analysis with the supervision of F. Bruno; drafting of the paper was mainly carried out by L. Vercellesi. All Authors state that they had complete access to the study data that support the publication.

\section{References}

1. Federfarma. Rapporto farmacie/abitanti in Italia [online]. Rome: Federfarma, 2012. http://www.federfarma.it/Farmacie-farmacie/Farmacie-e-farmacisti-in-Italia/La-presenza-dellefarmacie-sul-territorio.aspx (accessed 27 February 2012) [in Italian].

2. Martina A. La farmacia della comunicazione: il modello della comunicazione globale. Ed, Milano: GPAnet, 2003 [in Italian].

3. Rahim H, Shah B. Pharmacy students' perceptions and emotional responses to aggressive incidents in pharmacy practice. Am J Pharm Educ 2010; 74: 61.

4. Sporrong SK et al. 'We are white coats whirling round' - moral distress in Swedish pharmacies. Pharm World Sci 2005; 27: 223-229.

5. Berkowitz L. Frustration-aggression hypothesis: examination and reformulation. Psychol Bull 1989; 106: 59-73.

6. Chisholm-Burns MA et al. US pharmacists' effect as team members on patient care: systematic review and meta-analyses. Med Care 2010; 48: 923-933.

7. Bultman DC, Svarstad BL. Effects of pharmacist monitoring on patient satisfaction with antidepressant medication therapy. J Am Pharm Assoc (Wash) 2002; 42: 36-43.

8. Vegni E et al. Stories from doctors of patients with pain. A qualitative research on the physicians' perspective. Support Care Cancer 2005; 13: 18-25. 
9. Huggard P. Compassion fatigue: how much can I give? Med Educ 2003; 37: 163-164.

10. Eatough V, Smith JA. Interpretative phenomenological analysis. In: Willing C, Stainton-Rogers W, eds. Qualitative Research in Psychology. London: Sage, 2008: 179-194.

11. Cox K et al. An exploratory interview study of oncology patients and health care staff. Psychooncology 2007; 16: 985-993.

12. Duggan C, Bates I. Medicine information needs of patient: the relationships between information needs, diagnosis and disease. Qual Saf Health Care 2008; 17: 85-89.
13. Mead N, Bower P. Patient centeredness. A conceptual framework and review of the empirical literature. Soc Sci Med 2000; 51: 1087-1110.

14. Lonie JM. From counting and pouring to caring: the empathic developmental process of community pharmacists. Res Social Adm Pharm 2006; 2: 439-457.

15. Sanchez AM. Teaching patient-centered care to pharmacy students. Int J Clin Pharm 2011; 33: 55-57. 